\title{
Expectativas laborales de los egresados en Comunicación. Análisis de caso de los alumnos de Periodismo de la Universidad de Zaragoza
}

\section{Work Expectations of Graduates in Communication. Study-case of Journalism Students at University of Zaragoza}

\author{
María José Pérez Serrano. Universidad Complutense de Madrid (mariajoseperezserrano@pdi.ucm.es) \\ Carmen Marta Lazo. Universidad de Zaragoza (cmarta@unizar.es) \\ Miguel Ángel Ortiz Sobrino. Universidad Complutense de Madrid (maortiz@ucm.es)
}

Resumen:

Este trabajo aborda, desde la metodología cuantitativa, tres aspectos fundamentales para los estudios universitarios en Comunicación. Con la muestra correspondiente a los alumnos de cuarto de grado de Periodismo de la Universidad de Zaragoza del curso 2012-2013, se establecen conclusiones en torno a las expectativas de egresados en Comunicación, su formación universitaria y el mercado laboral. A partir de un aparato metodológico estadístico sencillo, se concluye que los estudiantes que eligieron los estudios de Periodismo de la UNIZAR lo hicieron fundamentalmente porque consideraban que "como periodistas podían contribuir a un mundo más justo", y, aunque piensan que podrían trabajar en un entorno multimedia, deben seguir formándose después de acabar el grado y no les será fácil ejercer la profesión periodística.

Palabras clave:

Ciencias de la Comunicación; expectativas laborales; Periodismo; EEES; Universidad de Zaragoza.

Abstract:

Using a quantitative methodology, this article tells about three fundamental aspects of university studies in the field of Communication. With a sample corresponding to the pupils of last year of the degree in Journalism of the University of Zaragoza (course 2012-2013), it establishes conclusions concerning the expectations of these graduates, their higher education and the current and upcoming labor market. This article concludes that these students chose the studies because they were thinking that "as journalists they could contribute to a better world". They considered that they might be employed at a multimedia environment and that they must continue to get updated instruction after graduating. Therefore, they agree that it will not be easy to become Journalism professional practitioners.

Keywords:

Communication Sciences; work expectations; Journalism; EHEA; University of Zaragoza. 


\section{Introducción}

La formación de ciudadanos libres a través de un sistema educativo de calidad es uno de los valores sobre los que se edifica la Unión Europea. Aunque la máxima no es nueva y su consecución no está exenta de dificultades, este planteamiento se ha traducido, en los últimos años, en el desarrollo del entramado educativo fruto del Espacio Europeo de Educación Superior (EEES).

En concreto, las líneas marcadas por la Declaración de Bolonia intentan desarrollar el cambio fundamental y necesario en las metodologías de enseñanza-aprendizaje asociado al cuarto objetivo de 1999, es decir, "la promoción de la cooperación Europea para asegurar un nivel de calidad para el desarrollo de criterios y metodologías comparables".

Este esfuerzo lo están desarrollando las Facultades dedicadas a las Ciencias de la Comunicación que, en las últimas cuatro décadas, según Murciano (2010: 6), "han consolidado en España un núcleo importante de conocimientos académicos teórico-prácticos y un saber hacer profesional en el Periodismo y en la comunicación, capaz de desarrollar e impulsar los modernos programas de reforma científico-pedagógica que requiere la formación universitaria en comunicación en la actualidad".

Aunque el "avance en la instalación y desarrollo del modelo de competencias, a partir de la implementación seria e imaginativa de múltiples proyectos" (Marta y Vadillo, 2013: 8) es una realidad, y el proceso de evaluación de las competencias adquiridas es una meta conseguida, aún queda campo analítico por escrutar en lo que se refiere al estudio de las sinergias existentes entre las expectativas de los estudiantes en Comunicación, la Universidad y el mercado laboral.

Como implicados directos en la relación con el alumno, desde el cuerpo docente de Ciencias de la Comunicación, se están haciendo esfuerzos por conocer y adecuarse a la sociedad que les circunda y a la actual realidad profesional.

\subsection{Límites, innovación y planteamiento}

Una de las bases conceptuales de este artículo está precisamente en sus límites. Dado que nuestra muestra (n=28) se circunscribe a los alumnos de cuarto de grado de Periodismo de la Universidad de Zaragoza del curso 2012-2013 es importante tener en cuenta que nos planteamos este aporte como el primer paso de una investigación ulterior que llevará la aplicación de esta misma metodología a otras universidades del resto del territorio nacional y contribuirá a valorar si la Universidad, en concreto los estudios de Comunicación, "está haciendo las cosas suficientemente bien" en lo que a transferencia de conocimiento y adecuación al mercado laboral se refiere (RQ).

Por lo tanto, el punto de partida se encuentra en el Plan de Estudios del Grado de Periodismo de dicha Universidad que fue aprobado mediante Resolución de la UNIZAR, de 10 de febrero de 2009 (BOA, 4 de marzo de 2009). Este Grado tiene, desde su puesta en marcha en el curso académico 2008-2009, un rasgo que le aporta singularidad ya que nació plena- 
mente adaptado al Espacio Europeo de Educación Superior. Por lo tanto, el conjunto de la muestra corresponde a la segunda promoción de Graduados en Periodismo de dicha Universidad.

En general, en el curso 2008-2009, momento en que los alumnos de nuestra muestra comenzaron sus estudios de grado, el número total de matriculados en Periodismo en España fue de 18.470 alumnos, lo que representa un 38\% sobre de los matriculados en Comunicación Audiovisual y Publicidad y Relaciones Públicas. Transcurridos los cuatro años del grado, en 2012, el número total de licenciados en Periodismo fue de 2.909.

Con todo ello, las hipótesis de partida para esta primera fase de la investigación son las siguientes:

H1. Los estudiantes de Periodismo son masivamente vocacionales y se enfrentan a los estudios en Comunicación con un alto grado de expectativas (variable EXP.) y motivación.

H2: Los alumnos de $4^{\circ}$ de grado de Periodismo de la Universidad de Zaragoza consideran que la Universidad (variable UNIV.) forma correctamente a los futuros comunicadores para trabajar en un entorno multimedia.

H3: Los futuros egresados en Periodismo piensan que la situación laboral no es buena y esto dificultará su inserción en el mercado de trabajo (variable MER. LAB.).

Cuadro 1: Modelo conjetural ${ }^{1}$ de la propuesta sobre expectativas laborales (EXP. LAB.)

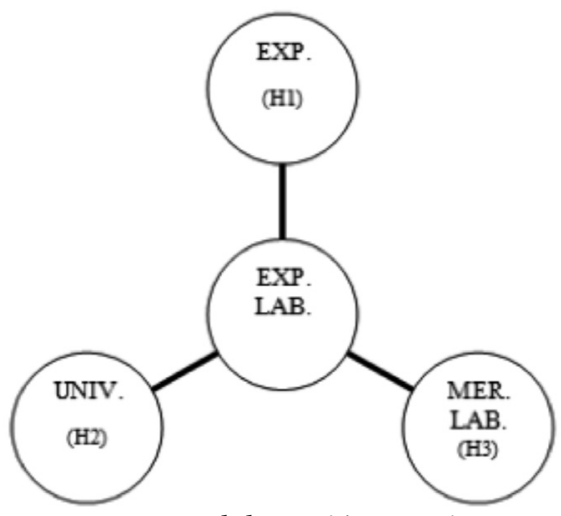

Fuente: Elaboración propia

Como paradigma a la hora de trabajar con el planteamiento, y la metodología cuantitativa (cuadros 1, 2 y 3), V. Berné, García y Orive, 2013, y Humanes y Roses, 2014. 


\section{Estado de la cuestión y metodología}

En la actual Sociedad de la Información, el alcance de esta función social de los periodistas se evidencia en el aumento anual del consumo mediático y, consecuentemente, en la aparición de nuevos medios informativos, como los canales de TDT (Televisión Digital Terrestre) o los periódicos digitales.

Ese creciente número de medios de comunicación abre las opciones de trabajo de los profesionales del Periodismo. Las exigencias consisten en una mayor calidad en la elaboración de productos mediáticos y mayor adaptabilidad a cada parte del proceso de producción informativo y a los nuevos soportes y recursos utilizados. La combinación del manejo de la tecnología con las habilidades periodísticas es una demanda que los medios actuales hacen a sus profesionales, ya que los soportes digitales exigen unos rasgos distintivos en la presentación de sus contenidos que los diferencien de los soportes tradicionales de información.

Si consideramos esta realidad, queda patente la importancia de estos estudios universitarios. El objetivo debe basarse en garantizar la formación de profesionales con una buena base teórica sin descuidar el desarrollo de las competencias del estudiante en el uso de los recursos y herramientas propias del ejercicio periodístico. Como señala De Miguel Díaz (2005: 17), “adaptarse a las características -nuevos conocimientos, patrones culturales, avances tecnológicos, valores dominantes, etc.- que rigen en este nuevo orden social implica un proceso de constante actualización para lo cual se exige a cada sujeto una capacitación personal crítica que favorezca la interpretación de la información circulante y la generación del conocimiento propio que le permita aprender de forma continua".

El Libro Blanco de los Títulos de Grado en Comunicación de la ANECA (2005) intentó poner de manifiesto la situación de este tipo de estudios y plantear una serie de orientaciones con el fin de aclarar hacia dónde debían tender estas titulaciones. El enfoque planteado no logró resolver uno de los mayores problemas que tradicionalmente ha afectado a los estudios de Periodismo, la distancia existente entre la academia y el campo profesional. Este aspecto se ha pretendiendo solventar con la modificación de planes de estudio, con el fin de lograr profesionales que se adapten a las nuevas necesidades del mercado laboral, aunque con una preparación académica que vaya mucho más allá que una simple formación profesional.

El perfil de los profesionales que demandan las empresas de comunicación ha cambiado notablemente en los últimos años fruto de algunos fenómenos que han hecho variar la realidad de los periodistas, tales como los ya señalados: creación de numerosos medios en la Red y el incremento en el número de canales. Pero no sólo se requiere del nuevo periodista un alto nivel de manejo tecnológico sino que hay que lograr profesionales capaces de elaborar esos contenidos de forma correcta y ética.

El manejo en el acceso a la información, el control de las fuentes y el dominio del uso correcto de la escritura son competencias que se sobreentienden adquiridas en el curso de la carrera universitaria. Pero vuelve a no ser suficiente, los grupos 
de comunicación buscan profesionales capaces de producir mensajes informativos en distintos soportes, lo que hace que el nuevo perfil varíe completamente del profesional del Periodismo en el que se han centrado hasta ahora gran parte de los estudios de comunicación. Ahora se demanda un periodista polivalente formado en todo tipo de medios y sistemas expresivos.

En la actualidad, los periodistas deben tener conocimiento de escritura, de diseño, de fotografía, de infografía, de radio, de televisión y de Internet. El periodista se convierte en un engranaje más dentro de la producción en cadena y la noticia se transforma en un producto. Desde la Universidad, hay que conseguir que los futuros periodistas puedan encajar en este modelo, pero hay que incidir en que no deben nunca renunciar a la profesionalidad, a la rigurosidad y al trabajo bien hecho vayan a insertarse en una gran empresa o vayan a asumir como emprendedores el desarrollo de un medio de nueva creación (y, para esto, la Universidad actual intenta dar respuesta, a través del aprendizaje basado en proyectos).

A su vez, las empresas consideran fundamental la capacidad de trabajo en equipo y la de someterse a una disciplina fundamentada en sus principios configuradores. En todo ello está el reto de los estudios universitarios de Comunicación y es ahí donde los docentes de los Grados de Periodismo tienen una importante labor.

En esta línea, en los últimos años han surgido análisis, investigaciones, estudios y aportaciones que, ya sea desde otras perspectivas del conocimiento como la Biblioteconomía y Documentación (Tejada, 2003) o la Psicología y la Motivación (Tuñez; Martínez y Abejón, 2010), o desde los estudios de Comunicación (Canel; Sánchez y Rodríguez; 2000; Farias, 2011; Humanes y Roses, 2014; Marta, 2009; Marta; Agustín y Ubieto, 2012; Ortiz, 2012; Osuna; Marta y Aparici, 2013; Sierra; Sotelo y Cabezuelo, 2010; Vadillo; Marta y Cabrera, 2010), abordan asuntos como las expectativas de los universitarios (Pichardo et al., 2007; o García, 2006) y su relación con el mercado laboral (Martín, 2012).

Una de esas investigaciones es la que tuvo como punto de partida la Summer Media School. En 2009 y en la Comunidad de Madrid, con motivo de la recomendación de la Unión Europea para que cada país profundizara en la alfabetización multimedia de sus niños y jóvenes, se llevó a cabo la primera edición de esta experiencia desarrollada por la Fundación FIDES y la Universidad Francisco de Vitoria, cuyo objetivo era el acercamiento de los futuros periodistas con el entorno universitario y la realidad profesional del Periodismo. A partir de ese momento y de manera anual, la Summer Media School es la base para un estudio pormenorizado sobre las expectativas de los futuros estudiantes de Comunicación.

\subsection{Método}

Spector (1982) señalaba que "quien estudie los resultados de las investigaciones de otros, debería conocer los principios de su diseño para ser capaz de emitir juicios razonables fundamentados sobre dichas investigaciones”. Para contribuir a que se cumpla esta sentencia, a continuación, desgranamos algunos aspectos particulares y esenciales de dicho diseño. 
Como de lo que se trataba era de medir el alcance y la intensidad de las expectativas laborales de los egresados en Periodismo e intentar, con ello, extrapolar y generalizar resultados, se consideró que la técnica más idónea era la encuesta. Más aún cuando ya se contaba con la experiencia previa de otras investigaciones en esta misma línea, tal como se ha visto en el apartado anterior con la Summer Media School.

En concreto, dicho estudio contó, desde comienzo de la década, con más de 700 participantes de entre 17 y 18 años, procedentes en su mayoría de la propia Comunidad de Madrid y también de otras comunidades como Valencia o Andalucía. Por sexos, la muestra respondió en las diferentes ediciones a la proporción habitual en estas carreras, es decir, mayoría de presencia femenina.

En esta investigación, que usa sencillas técnicas de estadística descriptiva, la población abarca a los alumnos del grado de Periodismo de la Universidad de Zaragoza y la muestra ocupa de manera exclusiva a los estudiantes de cuarto curso de dicho grado (V. Cuadro 2). Como precisión, se debe señalar que el retrato robot del encuestado (moda=M) es una mujer, de entre 20 y 25 años, que sólo se dedica a estudiar la carrera.

Cuadro 2: Características sociodemográficas de la muestra

\begin{tabular}{|c|c|c|}
\hline \multirow{2}{*}{ Sexo } & Hombre & $32,1 \%$ \\
\cline { 2 - 3 } & Mujer & $67,9 \%$ \\
\hline \multirow{4}{*}{ Edad } & Menos de 20 & $3,6 \%$ \\
\cline { 2 - 3 } & Entre 20-25 años & $78,6 \%$ \\
\cline { 2 - 3 } & Entre 25-30 años & $7,1 \%$ \\
\cline { 2 - 3 } & Entre 30-35 años & $3,6 \%$ \\
\cline { 2 - 3 } & Entre 40-50 años & $7,1 \%$ \\
\cline { 2 - 3 } & Más de 50 años & $0,0 \%$ \\
\hline \multirow{4}{*}{ Situación actual } & Sólo me dedico a estudiar la carrera & $42,8 \%$ \\
\cline { 2 - 3 } & Hago prácticas en medios & $28,6 \%$ \\
\cline { 2 - 3 } & Estoy trabajando en medios & $3,6 \%$ \\
\cline { 2 - 3 } & Trabajo en otro sector & $25,0 \%$ \\
\hline
\end{tabular}

Fuente: Elaboración propia

La obtención de información se consiguió a través de una encuesta autoadministrada durante el mes de mayo de 2013 y logró un trabajo de campo formado por 28 cuestionarios válidos. La encuesta contó con 25 preguntas con multirespuesta (Martín González, 2010:34-39). En alguna de ellas se brindaba al alumno la posibilidad de graduar su respuesta con esca- 
las Likert (la intensidad de la respuesta se marcaba con números del 1 al 5, siendo el 1 "nada"; el 2, "muy poco"; el 3, "algo"; el 4, "bastante", y el 5, "mucho").

Cuadro 3: Ficha técnica de la encuesta

\begin{tabular}{|l|l|}
\hline Ámbito geográfico & Zaragoza (Universidad de Zaragoza) \\
\hline Método recogida de la información & Cuestionario autoadministrado \\
\hline Tamaño muestral & 28 cuestionarios (válidos) \\
\hline Fecha del trabajo de campo & Mayo 2013 \\
\hline
\end{tabular}

Fuente: Elaboración propia

\section{Análisis y resultados}

A lo largo del trabajo de campo se insistió en una serie de ideas/ítems que permitieran extraer conclusiones acordes a los objetivos planteados en la investigación, entre los que, como ya se ha dicho, destacaban: expectativas, Universidad y mercado laboral. Si bien es cierto que el cuestionario contaba con 25 preguntas, para este artículo se han seleccionado para su análisis 17 de ellas, correspondientes a las hipótesis y variables propuestas.

Toca ahora mostrar cuáles han sido los resultados conseguidos. De su consecución, se pueden extraer conclusiones respecto al carácter que tiene este grupo de referencia como audiencia y como futuro profesional de los medios.

\subsection{Expectativas del futuro egresado en Periodismo}

Una de las primeras preocupaciones de los investigadores era conocer las motivaciones que llevaron a estos estudiantes a decantarse por los estudios de Periodismo. Al fin y al cabo, su elección supone la materia primigenia con la que se cuenta en las Facultades de Comunicación y son la base para las futuras expectativas. Además, extraer conclusiones sobre la vocación era importante porque permitía establecer el grado de conocimiento de los futuros alumnos sobre los medios de comunicación (Ortiz; Rodríguez y Pérez Serrano, 2011), y en qué medida no estaban condicionados en su elección por los aspectos menos "científicos" y más vinculados al efecto "moda" de la profesión. 
Cuadro 4: Pregunta núm. 4 “¿Por qué elegiste estudiar Periodismo?”

\begin{tabular}{|l|l|c|}
\hline & \multicolumn{1}{|l|}{} & VALORACIÓN MEDIA \\
\hline a) & Me gusta conocer otras culturas y países y esta es una profesión que me lo permitirá & 3,86 \\
\hline b) & Creo que como periodista puedo contribuir a un mundo más justo y mejor & 3,93 \\
\hline c) & Es una profesión nada rutinaria y muy atractiva & 3,71 \\
\hline d) & Es una profesión que me permitirá ser popular y trabajar con personajes populares & 2,07 \\
\hline e) & No podría dedicarme a otra cosa: es mi vocación & 2,61 \\
\hline f) & Es la profesión que me permitirá ganar el dinero suficiente para vivir como quiero. & 1,44 \\
\hline g) & Porque está de moda estudiar Periodismo & 1,07 \\
\hline h) & Porque es una carrera fácil & 1,36 \\
\hline i) & Porque he conocido a algunas personas que han estudiado esa carrera & 1,32 \\
\hline j) & Porque es una profesión que me permitirá influir en los demás y tener prestigio social & 1,50 \\
\hline k) & Porque hay muchas salidas profesionales para esta carrera & 1,75 \\
\hline l) & Porque mis padres y amigos me lo aconsejaron & 1,18 \\
\hline m) & Porque siempre me ha gustado escribir & 3,71 \\
\hline n) & Porque me atraía el plan de estudios & 2,61 \\
\hline o) & Porque admiro a los profesionales que ejercen esta profesión & 3,14 \\
\hline p) & Porque me gusta trabajar en equipo & 2,89 \\
\hline q) & Porque siempre he sido una persona creativa & 3,41 \\
\hline r) & Porque es la titulación a la que podía acceder con mi nota media & 1,78 \\
\hline s) & Porque es una profesión que siempre se ha ejercido en mi familia & 1,14 \\
\hline t) & Porque me han gustado siempre la información de actualidad & 3,67 \\
\hline u) & Porque quería conocer mejor el funcionamiento de las empresas de comunicación y los me- & 2,32 \\
\hline v) & Pios & \\
\hline
\end{tabular}

Fuente: Elaboración propia

Como pone de manifiesto la valoración media (promedio $=\overline{\mathrm{X}}$ ) de las motivaciones propuestas, la vocación de los futuros comunicadores tiene cierta consistencia y se aleja de un planteamiento idealizado de la profesión. De hecho, las dimensiones sociales o éticas del Periodismo se sitúan como razones de peso para la elección de los estudios de Periodismo, así como que a los estudiantes les interesen los contenidos de los medios. 
Dentro del grupo de ítems vinculados a la motivación para elegir una titulación en Comunicación, destaca que apenas tienen incidencia los vinculados a los motivos de logro y prestigio. Los futuros estudiantes de Comunicación declaran que el dinero que puedan ganar, las salidas profesionales, el prestigio que puedan alcanzar o el efecto "moda" no resultan para ellos lo más determinante.

Cuadro 5: Representación gráfica de las valoraciones medias sobre las motivaciones

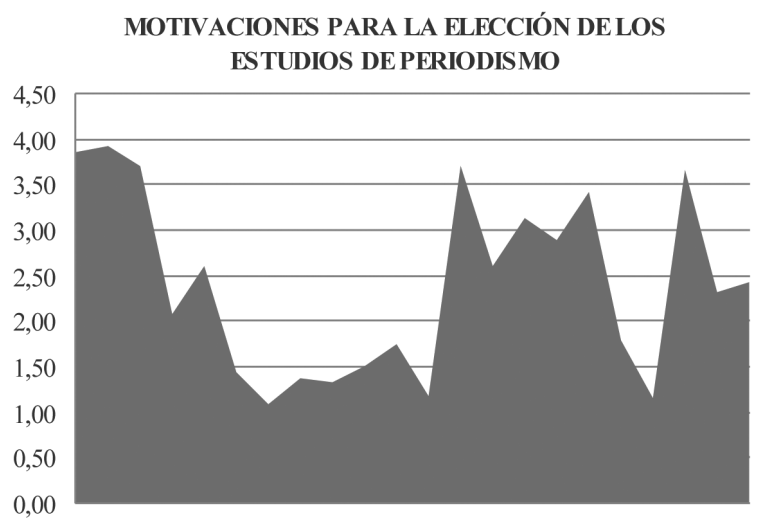

a) b) c) d) e) f) g) h) i) j) k) l) m) n) o) p) q) r) s) t) u) v)

Fuente: Elaboración propia

En líneas generales, sin estar todavía inmersos en el día a día de la práctica profesional de la comunicación atisban un mañana donde seguro van a tener que brillar por su talento y su preparación para hacer frente y encontrar su lugar en un mercado competitivo y difícil. Por eso, el $60 \%$ de los encuestados consideran que, antes de enfrentarse a ese momento, deben seguir formándose a través de posgrados, otras carreras e idiomas y no hay ningún alumno que no se haya planteado qué va a hacer después de su paso por las aulas. 


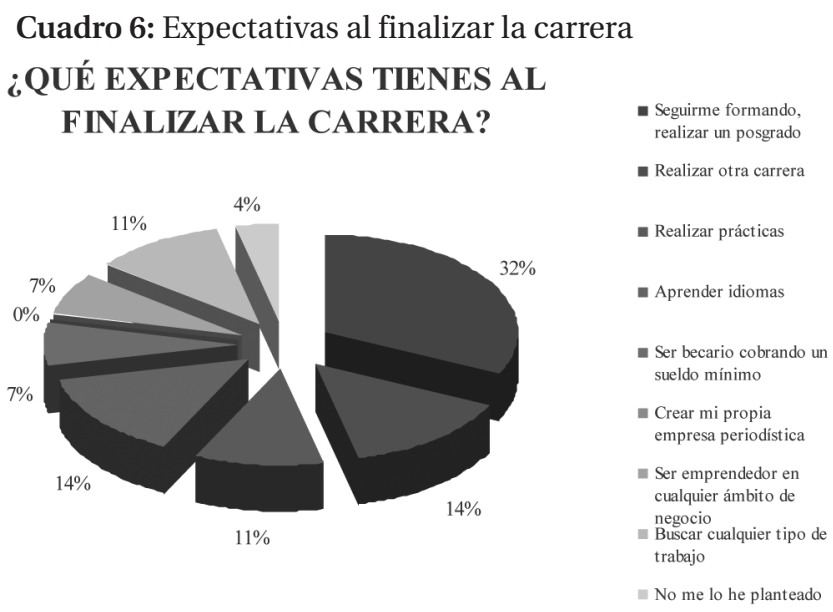

Fuente: Elaboración propia

Los datos extraídos en este análisis arrojan como resultado que, en general, a los estudiantes de la Universidad de Zaragoza les gustaría llegar a trabajar en los medios tradicionales e Internet. En concreto, aproximadamente un tercio de ellos piensan que les gustaría trabajar en varios medios a la misma vez.

Cuadro 7: Áreas de interés para la futura dedicación profesional

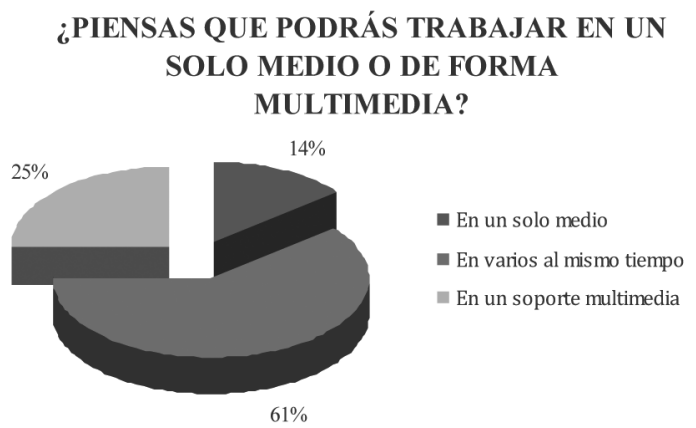

Fuente: Elaboración propia

Lo que coincide ampliamente con que, al ser preguntados de forma específica, sobre si creen que podrán trabajar en varios medios a la misma vez, el $61 \%$ considera que sí. Como puede observarse, respecto a las áreas del Periodismo donde les gustaría emplearse, los datos recogidos apuntan a que la tendencia mayoritaria es el ámbito de la "cultura", seguida por 
la información “internacional”. Lo que, en una correlación sencilla, refuta la idea de que de promedio 3,86 puntos, señalan que una de las razones por las que estudían Periodismo es porque "les gusta conocer otras culturas y países y esta es una profesión que se lo permitirá.

Cuadro 8: Áreas de interés para la futura dedicación profesional
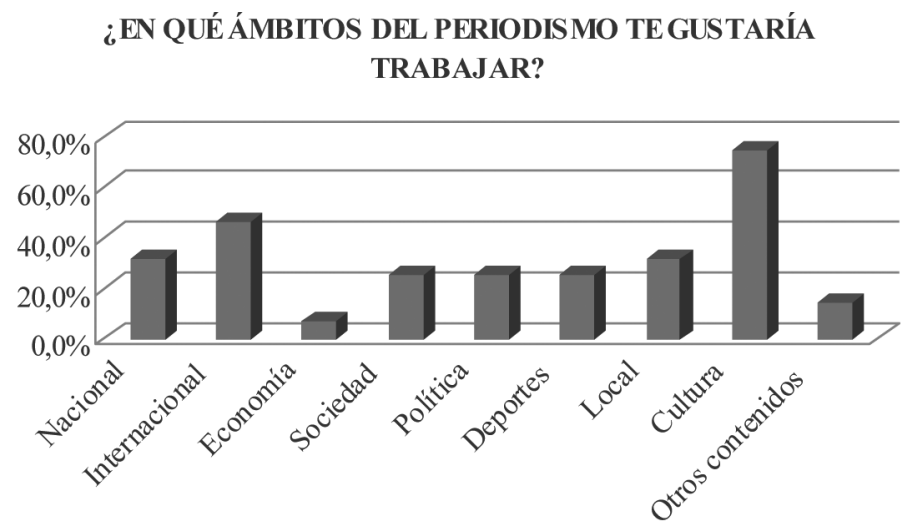

\section{¿PIENSAS QUE PODRÁS TRABAJAR EN UN \\ SOLO MEDIO O DE FORMA \\ MULTIMEDIA?}

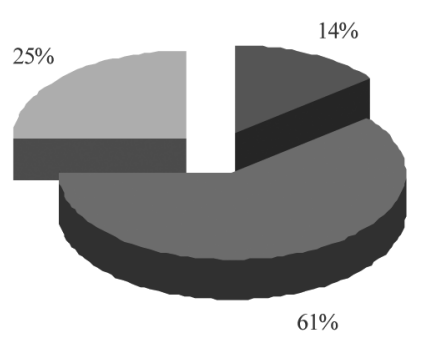

En un solo medio

- En varios al mismo tiempo

En un soporte multimedia

Fuente: Elaboración propia

\subsection{Percepción sobre la formación universitaria en Periodismo}

Por otro lado, importaba extraer datos sobre la perspectiva que alumnos tenían sobre formación universitaria, sobre todo en aquellos aspectos que podrían indicar si ven que su formación debe ir encaminada a la dimensión más práctica, es decir al oficio del Periodismo, o desde el punto de vista más deontológico y científico. En este sentido, aunque son conscientes de cuál ha sido el desarrollo de su grado y de que, según ellos, los pilares de su formación deberían estar en "la 
combinación de una buena teoría con la práctica” y “tener profesionales en activo entre sus profesores”, el 71\% de los encuestados consideraron que realizar prácticas previas resulta ser "bastante" o "muy" importante para conseguir trabajo al terminar la carrera (la valoración media de esta pregunta fue de 3,79).

Cuadro 9: Pregunta núm. 19

“¿Qué debería primar en la enseñanza del Periodismo en la Universidad?”

\begin{tabular}{|l|l|c|}
\hline & \multicolumn{1}{|c|}{} & VALORACIÓN MEDIA \\
\hline a) & La dimensión práctica de la carrera & $14,3 \%$ \\
\hline b) & La combinación de una buena teoría con la práctica & $28,6 \%$ \\
\hline c) & $\begin{array}{l}\text { La formación teórica del alumno que le permita valorar y contextualizar bien la informa- } \\
\text { ción ya que la práctica (y la tecnología aplicada) es fácil y rápida de aprender }\end{array}$ & $14,3 \%$ \\
\hline d) & Una buena relación con las empresas de comunicación a través de convenios de prácticas. & $14,3 \%$ \\
\hline e) & Tener una buena tecnología para realizar prácticas & $0,0 \%$ \\
\hline f) & Tener profesionales en activo entre sus profesores & $28,6 \%$ \\
\hline
\end{tabular}

Fuente: Elaboración propia

Asimismo, se puede concluir que estos estudiantes de Ciencias de la Comunicación no tienen una imagen excesivamente positiva sobre su formación. De hecho, al preguntarles si pensaban que la Universidad les había formado bien como periodistas, el 60\% de ellos respondían que "nada" o "poco". Lo que traducido a la media de satisfacción en esta pregunta el nivel se colocaba en un 2,29 (en la escala 1-5). Su percepción sobre el EEES no difiere en demasía de los datos antepuestos. Ya que la valoración media que los alumnos tienen sobre el proceso de reforma de la Universidad española en su adaptación al Espacio Europeo de Educación Superior (Bolonia) no llega al aprobado (situándose en un 2,43 sobre cinco). Además, el $57 \%$ considera que solo algunas universidades públicas y privadas forman correctamente a los futuros periodistas. 
Cuadro 10: Pregunta núm. 17

¿CREES QUE LA UNIVERSIDAD, EN GENERAL, FORMA BIEN A LOS FUTUROS PERIODISTAS?

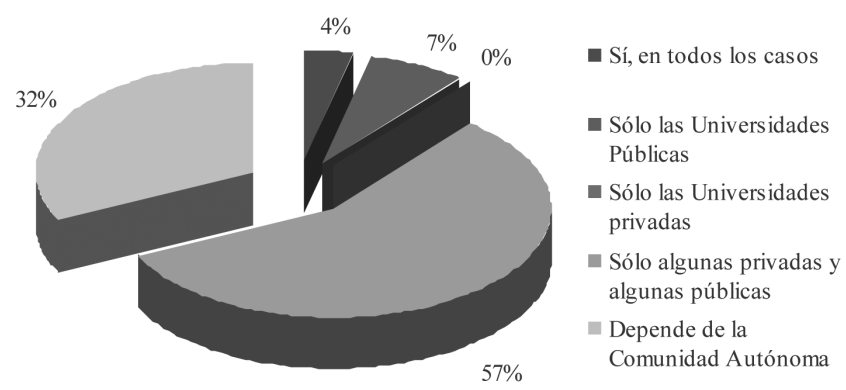

Fuente: Elaboración propia

\subsection{Consideración en torno a la inserción del futuro periodista en el mercado laboral}

La realidad profesional del sector de la Comunicación se caracteriza por "el intrusismo laboral, la baja remuneración, el paro, la inestabilidad laboral y la incompatibilidad con la vida familiar" (Farias Batlle, 2010: 15). Cuando se le preguntó al estudiante de Periodismo de la UNIZAR “en qué medida pensaba que sería fácil su acceso al mercado laboral", la respuesta fue contundente: el 96\% consideró que iba a ser "poco" o "nada" fácil incorporarse a un trabajo (y la media -promedio= B de la respuesta se colocó en un 1,64 sobre 5) y las razones que esgrimían para ello seguían el siguiente parámetro.

Cuadro 11: Pregunta núm. 22

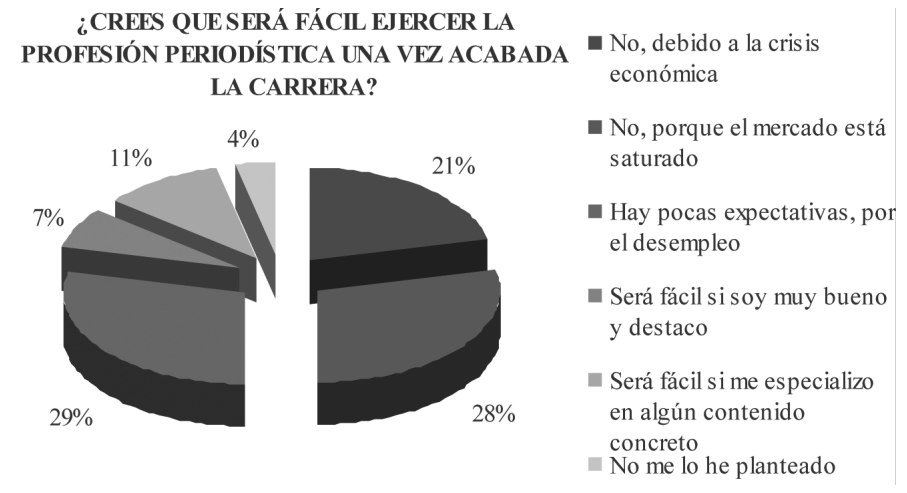

Fuente: Elaboración propia 
Aunque el 39\% de los encuestados no se han planteado dónde creen que van a encontrar trabajo, de los que sí lo han hecho el $47 \%$ cree que su horizonte laboral está, como mucho, en Europa. Un ejemplo paradigmático lo podemos encontrar en la respuestas vinculadas a la comunidad autónoma, el $7 \%$ cree que encontrará trabajo en la suya propia, aunque al $40 \%$ le gustaría trabajar en ella.

Cuadro 12: Preguntas núm. 24 y 25

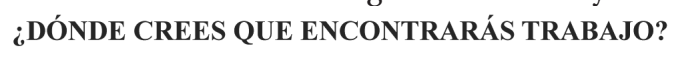

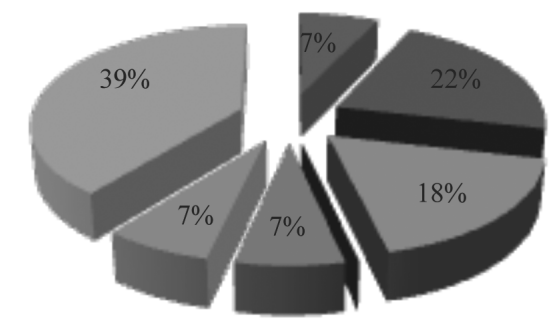

$0 \%$

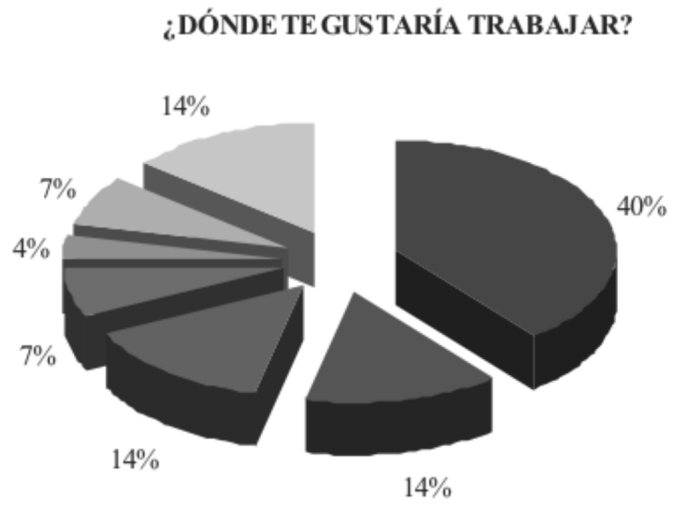

Fuente: Elaboración propia

\author{
- En tu Comunidad \\ Autónoma \\ - En tu país \\ - En Europa \\ - En EEUU \\ En Latinoamérica \\ "En otros países \\ No me lo he planteado
}

- En tu Comunidad
Autónoma
En tu país
- En Europa
- En EEUU
- En Latinoamérica
- En otros países
" No me lo he planteado

\section{Conclusiones}

Si se hace un planteamiento correlacional entre las hipótesis y los resultados obtenidos, podemos decir que las H1 (Los estudiantes de Periodismo son masivamente vocacionales y se enfrentan a los estudios en Comunicación con un alto grado 
de expectativas y motivación) y H3 (los futuros egresados en Periodismo piensan que la situación laboral no es buena y esto dificultará su inserción en el mercado de trabajo) se han corroborado, pero no ha sucedido lo mismo con la H2 (Los alumnos de $4^{\circ}$ de grado de Periodismo de la Universidad de Zaragoza consideran que la Universidad forma correctamente a los futuros comunicadores para trabajar en un entorno multimedia).

Estos correlatos plantean dos vías de actuación importantes que abren las posibilidades de esta investigación. Por un lado, en lo que se refiere al ampliar el campo analítico al resto de Facultades y grados en Periodismo y, por otro, a mejorar, en la medida de las posibilidades, el grado de satisfacción de los estudiantes de Periodismo, lo que redundará en la mejora de la imagen de marca de la Universidad y será síntoma de que no solo se hacen las cosas bien (como no ponemos en duda que se hacen), sino que los alumnos así lo perciben.

\section{Referencias bibliográficas}

Berné Manero, C., García Uceda, M. E. y Orive Serrano, V. (2013): “Determinantes del comportamiento de consumo de televisión: un modelo estructural”, Revista Mediterránea de Comunicación, vol. 4, n. 2, pp. 209-224.

Canel, M. J.; Sánchez, J. J. y Rodríguez, R. (2000): Periodistas al descubierto. Retrato de los profesionales de la información. Madrid: Centro de Investigaciones Sociológicas.

De Miguel Díaz, M. (2005): “Cambio de paradigma metodológico en la Educación Superior. Exigencias que conlleva”, Cuadernos de Integración Europea, n. 2, pp. 16-27.

Farias Batlle, P. (2010): “La credibilidad, el mejor salvavidas”, en Farias Batlle, P. (dir.): Informe Anual de la Profesión Periodística 2010. Madrid: APM, pp. 15-19.

- (dir.) (2011): Informe anual de la profesión periodística. Madrid: APM.

Garcia Garduño, J. M. (2006): “Motivación y expectativas para ingresar en la carrera de profesor de educación primaria: un estudio de tres generaciones de estudiantes normalistas mexicanos de primer ingreso", REDIE: Revista de Investigación Educativa, vol. 8, n. 2, pp. 1-19.

Humanes, M. L. y Roses, S. (2014): “Valoración de los estudiantes sobre la enseñanza del Periodismo en España”, Comunicar, n. 42, 181-188.

Marta Lazo, C. (2009): “El proceso de reconversión de la comunicación en el EEES: Presentación”, Revista Icono 14, n. 14, pp. 1-7.

Marta Lazo, C. y Vadillo Bengoa, N. (coord.) (2013): Evaluación de la implantación del EEES en los estudios de Comunicación. La Laguna: Cuadernos Artesanos de Latina (34).

Marta Lazo, C., Agustín Lacruz, M. C. y Ubieto Artur, M. I. (coords.) (2012): Competencias interdisciplinares para la comunicación y la información en la sociedad digital. Madrid: Icono 14. 
Martín Bernal, O. (2012): "Retratos robot del periodista en el entorno digital. Los nuevos perfiles profesionales y laborales", en Diezhandino, M. del (coord.): El Periodista en la encrucijada. Barcelona: Ariel, pp. 79-111.

Martín González, J. A. (2010): “Cinco consejos para la investigación cuantitativa”, Marketing+Ventas, n. 258 [Consultado el 09/03/14].

Murciano, M. (2010): “La transformación de los estudios de comunicación en España Nuevo contexto europeo de los estudios universitarios y enseñanzas para favorecer un espacio de cooperación iberoamericano”, Diálogos de la Comunicación, n. 79, pp. 11-10.

Ortiz Sobrino, M. Á. (2012): "Principales señas de identidad de los estudiantes de Comunicación en el EEES de la Comunidad de Madrid en 2012: expectativas, perfil de opción y relaciones mediáticas”, Estudios sobre el mensaje periodístico, vol. 18, pp. 661-670.

Ortiz Sobrino, M. Á., Rodríguez Barba, D. y Pérez Serrano, M. J. (2011): “Perfil de entrada de los futuros alumnos de Comunicación en las universidades de Madrid, en 2010/2012: la reafirmación de los rasgos”, Revista de la SEECI (Sociedad Española de Estudios de la Comunicación Iberoamericana), n. 26, pp. 1-21.

Osuna Acedo, S.; Marta Lazo, C. y Aparici Marino, R. (2013): "Valores de la formación universitaria de los comunicadores en la sociedad digital: más allá del aprendizaje tecnológico, hacia un modelo educomunicativo”, Razón y Palabra, n. 81, pp. $1-33$.

Peinado Miguel, F. (2011): "Motivación + Innovación + Bolonia = Formación Superior Necesaria”, Anuario de la Comunicación 2011, pp. 114-115.

Pichardo, M. C.; García, A. B.; Fuente, J. de la y Justicia, F. (2007): "Estudio de las expectativas en la Universidad: análisis de trabajos empíricos y futuras líneas de investigación”, REDIE: Revista electrónica de investigación educativa, vol. 9, pp. 1-17.

Sierra, J.; Sotelo, J. y Cabezuelo, F. (2010): “Competencias profesionales y empleo del futuro periodista: el caso de los estudiantes de Periodismo de la UCH-CEU”, @tic, n. 5, pp. 8-19.

Spector, P. E. (1982): Research design. Beverly Hills: Sage Pub.

Tejada, C. M. (2003): "Perfil, motivaciones y expectativas de los alumnos de primer curso de la escuela de Biblioteconomía y Documentación de la UCM”, Revista General de Información y Documentación, vol. 13, n. 1, pp. 195-211.

Tuñez López, M.; Martínez Solana, Y. y Abejón Mendoza, P. (2010): “Nuevos entornos, nuevas demandas, nuevos periodistas", Estudios sobre el Mensaje Periodístico, n. 16, pp. 79-94.

Vadillo, N.; Marta Lazo, C. y Cabrera, D. H. (2010): “Proceso de adaptación de los estudios de Comunicación al EEES. El caso de Aragón, una comunidad pionera”, Revista Latina de Comunicación Social, n. 65, pp. 187-203. 